www.nature.com/clinicalpractice/uro

\title{
The influence of the internet on patients' expectations
}

\author{
Alex F Broom
}

Patients are now able to access a huge amount of information about their health via the internet. Advances in information technology have the potential to radically transform both decisionmaking and treatment processes. The rise in popularity of the internet has, however, been accompanied by claims that it could potentially harm patients and the doctor-patient relationship. In particular, it has been criticized for turning patients away from conventional treatments, misleading patients as to the efficacy of alternative therapies, unnecessarily increasing consultation times, and creating conflict between the doctor and patient in medical consultations. Until recently there has been little research examining the veracity of such claims, but research in the social sciences is beginning to break down such oversimplified views of the effects of the internet and the internet-informed patient.

We are now seeing the positive effects of the internet. Many patients are reporting increased awareness of the range of treatment options available, improved certainty in the decisionmaking and treatment processes and an increased sense of control resulting from the opportunity to be actively involved throughout the disease process. It would seem that, rather than encouraging patients to seek alternative therapies, information from the internet may actually help to clarify the subtleties involved in particular interventions, thereby increasing levels of concordance. It also seems likely that the value of the internet lies in its role as a source of information that can provide some redress to traditional imbalances in knowledge and power in doctor-patient encounters.

Although it is important to acknowledge the potential benefits of the internet for patients, it is vital not to overestimate them. This point is illustrated by the fact that patients frequently have negative experiences of the internet, with many patients lacking confidence in their ability to seek out and assess information. For some patients the internet may introduce yet another source of anxiety, when they merely desire their specialist
.... the value of

the internet

lies in its role

as a source of

information

that can

provide some

redress to

traditional

imbalances

in knowledge

and power in

doctor-patient

encounters.

AF Broom is a

Postdoctoral Research

Fellow based in the

School of Social

Sciences, University

of Queensland,

Australia.

\section{Competing interests}

The author declared he has

no competing interests

www.nature.com/clinicalpractice doi:10.1038/ncpuro0432 to take control of decision-making processes. Determining how involved a patient wants to be in directing their management is quite difficult and, unfortunately, is all too often determined by demographics (e.g. socioeconomic status) rather than the patient's preference; a problem seen in the experiences of prostate cancer patients. Moreover, the notion of reducing patient anxiety is sometimes used by specialists as a justification for reinforcing paternalistic dynamics.

Medical specialists differ in their views of, and responses to, internet-informed patients. Some describe significant improvements in decision-making processes, increased patient autonomy, satisfaction with decision making, shared responsibility and increased concordance. Others, however, feel negative toward internet-informed patients and report conflictual dynamics within medical consultations. Clearly there is something of a split between those specialists prepared to move toward a more open and consultative approach to decision-making processes and the doctor-patient relationship, and those who seek to preserve a more paternalistic dynamic within medical consultations. The latter approach merely alienates many patients and results in lower levels of satisfaction with care. This is unfortunate given that, when utilized effectively, the internet can provide a useful addition to existing patient information and support services.

Most patients desire (and will appreciate) guidance from their specialist regarding how best to use the internet, and will readily accept reasonable explanations as to the inappropriateness of information they have retrieved. It is important that doctors acknowledge the efforts of their patients and encourage their 'activeness' in treatment processes, as well as ensuring they are not misled as to the efficacy of treatments. Dismissing patients' online information retrieval, regardless of how inappropriate the material they bring along, will only service to alienate them and potentially result in them utilizing inappropriate information. 